\title{
Transcriptomic characterization of the dorsal lobes after hepatectomy of the ventral lobe in zebrafish
}

\author{
Guohui Feng ${ }^{1,2}$, Yong Long ${ }^{1}$, Jinrong Peng ${ }^{3}$, Qing Li ${ }^{1^{*}}$ and Zongbin Cui ${ }^{{ }^{*}}$
}

\begin{abstract}
Background: The liver possesses an ability of compensatory growth after removing three of five lobes in mammals or one of three lobes in zebrafish. The reenter of hepatocytes into the cell cycle is one of the hallmarks for the initiation of liver compensatory growth, but cellular and molecular mechanisms underlying the activation of hepatocytes remain largely unknown.

Results: To better understand the process, transcriptional profiles of the remaining liver dorsal lobes in female zebrafish were generated with RNA-seq. About 44 million raw reads were obtained from three sequencing libraries and $71 \%$ of raw reads were mapped to the reference genome of zebrafish. A total number of 5652 genes were differentially expressed in at least one of two time points during the compensatory growth of liver dorsal lobes and classified into different functional categories. A number of genes encoding angiogenesis-related growth factors/ligands and apoptosis-associated cytokines were strongly expressed at 6-h time point after the removal of the ventral lobe. Gene ontology enrichment analysis of genes up-regulated during early stages of liver compensatory growth revealed that small GTPase-mediated signal transduction, RNA processing and intracellular protein transport were the most highly overrepresented biological processes and SNARE interactions in vesicular transport, proteasome and basal transcription factors were the most highly enriched pathways. Moreover, 477 genes differently expressed during liver compensatory growth of both female zebrafish and mice were involved in the response to stimulus, DNA replication, metabolic processes of fatty acid, lipid and steroid, multicellular organismal homeostasis and extracellular matrix constituent secretion.
\end{abstract}

Conclusions: Multiple biological processes and signaling pathways are immediately activated in remaining dorsal lobes of female zebrafish right after removal of the ventral lobe and these findings provide crucial clues for further identification of cis-elements and trans-factors that are extensively involved in the initiation of liver compensatory growth.

Keywords: Zebrafish, Hepatectomy, Liver compensatory growth, RNA-seq

\section{Background}

The liver of vertebrates from fish to humans possesses a phenomenal capacity of compensatory growth and/or regeneration even after massive tissue loss [1-3]. This process is hallmarked by the reenter the cell cycle of hepatocytes, which allows liver to recover the lost mass without jeopardizing the viability of entire organism $[4,5]$.

\footnotetext{
*Correspondence: qli@ihb.ac.cn; zbcui@ihb.ac.cn

${ }^{1}$ The Key Laboratory of Aquatic Biodiversity and Conservation of Chinese Academy of Sciences, Institute of Hydrobiology, Chinese Academy of Sciences, Wuhan 430072 Hubei, China

Full list of author information is available at the end of the article
}

Nevertheless, the ability of compensatory growth and/or regeneration is greatly compromised after being damaged by cirrhosis and hepatitis [6]. Therefore, it is indispensable to understand molecular mechanisms underlying the compensatory growth and/or regeneration of liver for improving treatments of liver diseases.

Partial hepatectomy $(\mathrm{PH})$ by removing three of five liver lobes is widely utilized for studies of liver compensatory growth and regeneration in mammals $[2,7]$. The liver of adult zebrafish consists of one ventral lobe and two dorsal lobes, which are quite different with those in mammals [8]. The removal of two liver lobes including 
the ventral lobe and either the left or right dorsal lobe often leads to strong bleeding and lower survivals, so the analysis of liver compensatory growth in zebrafish is usually performed through a surgical procedure of removing the whole ventral lobe $[6,9]$ and such damaged liver can enter a tightly regulated regeneration process to restore the lost mass and vital functions [10], but the ablated liver lobes never grow back and the original liver mass and function are reached by compensatory growth of the remaining liver lobes. It is suggested that this process is synchronously and precisely controlled by proliferative responses of differentiated somatic cells, including hepatocytes that appear to be mainly responsible for this course [2, 11, 12], liver sinusoidal endothelial cells (LSECs) [13-15], Kupffer cells [16] and hepatic stellate cells (HSCs) [17]. Extensive studies in rodents have uncovered the crucial roles of some cytokines, growth factors, microRNAs, and molecular events such as matrix remodeling and metabolic signals, in regulating this complex process $[1,10,18-20]$. However, cellular and molecular events that trigger the compensatory growth and/or regeneration of remaining liver after $\mathrm{PH}$ remain largely unclear.

Zebrafish were previously used to study molecular mechanisms of inner retinal neurons regeneration [21], heart regeneration [22], liver regeneration [6] and human diseases as a complement to mouse models [23]. Transcriptomic analysis of the liver compensatory growth in rodents has been performed by using the microarray [24-27], but gene expression profiles of liver compensatory growth in zebrafish remain to be characterized. Additionally, RNA-seq appears to be more powerful than the microarray for the identification of molecular events due to its high sensitivity and accuracy, digital expression and the ability to distinguish transcript isoforms [28].

In this study, we aim to characterize the transcriptional responses in cells of remaining dorsal liver lobes to $\mathrm{PH}$ using RNA-seq and uncover factors and events that are crucial for the compensatory growth of dorsal lobes after the removal of whole ventral lobe in adult female zebrafish.

\section{Results}

RNA-seq analysis of remaining dorsal lobes after hepatectomy of the ventral lobe

To identify molecular signals for the initiation of liver compensatory growth and/or regeneration, gene expression profiles of dorsal lobes in female zebrafish were analyzed with RNA-seq at 6-h and 24-h time points after removing the whole ventral lobe (Fig. 1a). High-throughput sequencing generated approximately $15.6 \mathrm{M}$ of total reads from sham-treated livers, $13.18 \mathrm{M}$ of total reads from the 6-h time point after $\mathrm{PH}$ and $15.27 \mathrm{M}$ of total reads from the 24-h time point after PH (Table 1) and $72.18 \%, 68.51 \%$ and $71.77 \%$ of these total reads were successfully mapped to the reference genome, respectively. The total number of mapping events generated by TopHat was $14.54-20.53 \mathrm{M}$. The read number of potential splice variants was 1.91$3.28 \mathrm{M}$, representing $13.05-15.98 \%$ of the total alignment (Table 1).

After read mapping, gene expression was calculated using Cufflinks. As described previously [29, 30], genes with a mean abundance $>0.1$ FPKM (Fragments per kilobase of transcript per million fragments mapped) were included in the analysis. A total of 13,470 genes in sham-treated dorsal lobes, 13,111 genes in remaining dorsal lobes at 6-h time point and 13,414 genes at 24-h time point after hepatectomy of the ventral lobe were used for further analysis (Table 1).

\section{Genes differentially expressed during the compensatory growth of liver dorsal lobes}

Genes differentially expressed during early stages of liver compensatory growth after hepatectomy of the ventral lobe were listed in Additional file 1. In comparison with those in sham-treated livers, 3967 and 3401 genes differentially expressed in regrowing dorsal lobes at 6-h and 24-h time points after $\mathrm{PH}$ were found, respectively (Fig. 1b) and 1716 genes were found to be differentially expressed at both 6-h and 24-h time points during liver compensatory growth after hepatectomy of the ventral lobe. The numbers of up- and down-regulated genes were 2245 and 1722 at 6-h time point and 1631 and 1770 at 24-h time point after PH (Fig. 1b).

To classify the dynamic transcriptome in early regrowing dorsal lobes of zebrafish liver on a global scale, we performed of gene expression profile clustering. The 5652 gene transcripts that are differentially expressed in at least one of the two time points during the compensatory growth process were categorized into 8 different clusters according to the similarity of their expression patterns (Additional file 1; Fig. 1b and c). Among these genes, 1264 (22.4\%, cluster I) and 670 genes (11.9\%, cluster II) were specifically up-regulated at 6-h time point and 24-h time point after hepatectomy of the ventral lobe, respectively; however, 71 genes (1.3\%, cluster III) were up-regulated at 6-h time point but down-regulated at 24-h time point after $\mathrm{PH}$. Moreover, 910 genes (16.1\%, cluster IV) were continuously up-regulated during the process of liver compensatory growth. Likewise, 987 (17.5\%, cluster V) and 1015 genes $(18.0 \%$, cluster VI) were specifically down-regulated at 6-h time point and 24-h time point after $\mathrm{PH}$, respectively. There are 51 genes $(0.9 \%$, cluster VII $)$ downregulated at 6-h time point but up-regulated at 24-h time point after removing the ventral lobe and 684 genes (12.1\%, cluster VIII) continuously down-regulated during the process of liver compensatory growth process. Cav1 (caveolin 1) and egfr (epidermal growth factor receptor), which have been shown to be required for liver 

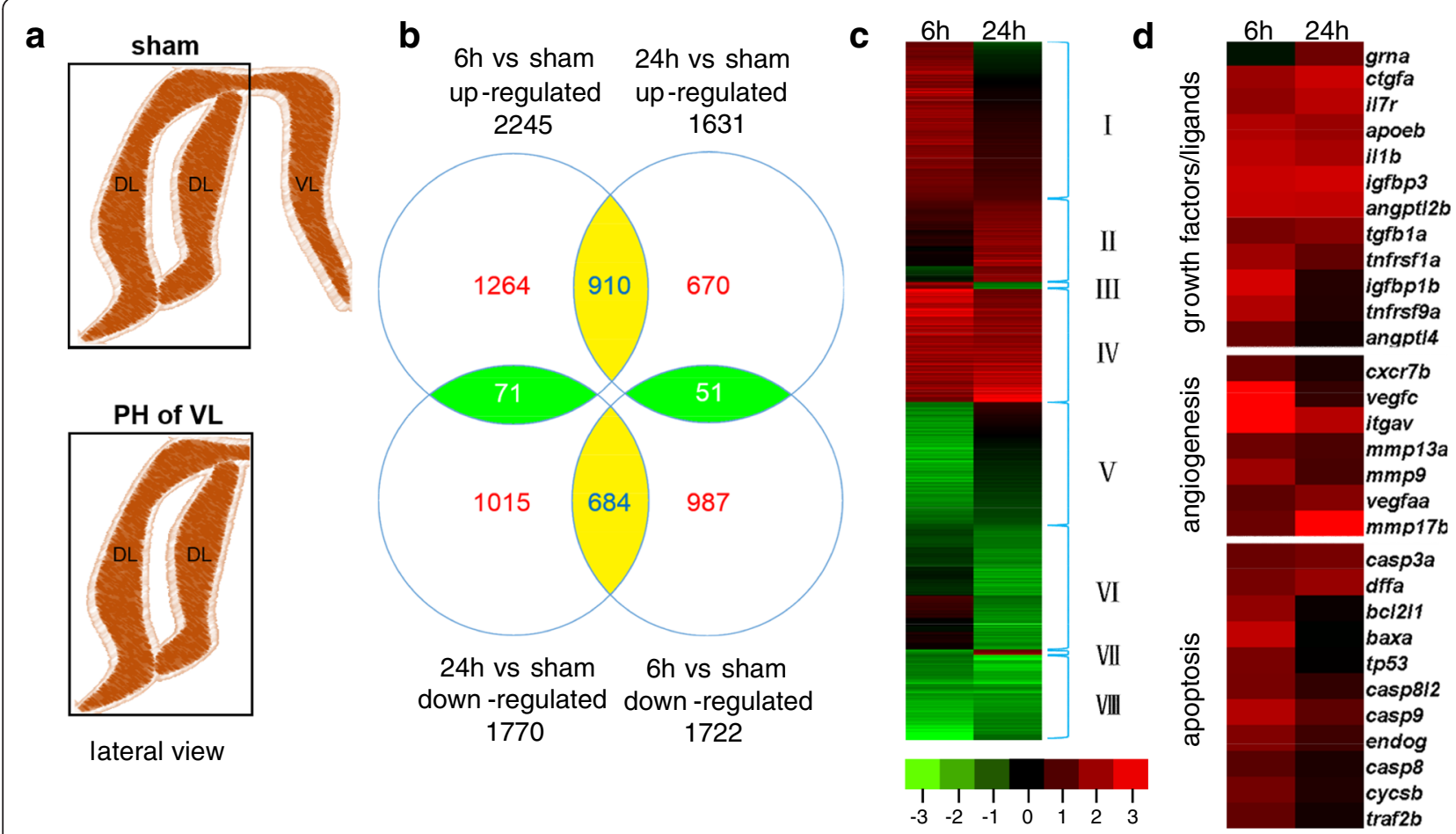

Fig. 1 Gene expression profiling of liver compensatory growth in zebrafish. a Schematic diagram of sham surgery and hepatectomy of the whole ventral lobe and materials collection in sham and experiment groups. DL: dorsal lobe, VL: ventral lobe, PH of VL: partial hepatectomy of the whole ventral lobe. Black box indicates the materials collected in correspondent groups. $\mathbf{b}$ Venn diagrams represent the number of differentially expressed genes during the liver compensatory growth. c Gene clustering based on gene expression profiles across the compensatory growth process. A total of 5652 genes are differentially expressed during this process. These genes were clustered into eight groups based on the similarity of their expression patterns during liver compensatory growth at 6-h time point and 24-h time point after removal of the ventral lobe. $\mathbf{d}$ Genes encoding growth factors/ligands and involved in angiogenesis or apoptosis were clustered based on their expression patterns at 6-h time point and 24-h time point after surgery. The gene name, gene symbol, and log2 fold change of each gene can be found in Additional file 1. The color chart indicating fold change of expression uses a base 2-logarithm scale. Red and green represent increased and decreased gene expression, respectively

compensatory growth in mice by genetic analysis [31-33], were identified in cluster IV and cluster I, respectively.

In addition, several of genes including apoeb (apolipoprotein $\mathrm{Eb}$ ), il1b (interleukin 1, beta), igfbp3 (insulin-like growth factor binding protein 3), igfbp1b (insulin-like growth factor binding protein $1 \mathrm{~b}$ ), vegfc (vascular endothelial growth factor c), itgav (integrin, alpha V), baxa (bcl2associated X protein, a) and casp9 (caspase 9), were found to be strongly expressed at 6-h time point after $\mathrm{PH}$ (Additional file 2 and Fig. 1d).

Table 1 Statistics for the mapping of reads

\begin{tabular}{llll}
\hline Sample name & sham & $6 \mathrm{~h}$ & $24 \mathrm{~h}$ \\
\hline Total reads (M) & 15.60 & 13.18 & 15.27 \\
Mapped reads (M) & 11.26 & 9.03 & 10.96 \\
\% of mapped & 72.18 & 68.51 & 71.77 \\
Total alignment (M) & 20.53 & 14.54 & 15.94 \\
Total potential splice variants (M) & 3.28 & 1.91 & 2.08 \\
Included genes & 13,470 & 13,111 & 13,414 \\
\hline
\end{tabular}

\section{Validation of RNA-seq data by qPCR}

The expression of 15 genes from cluster III, IV and VII was selected to be measured by qPCR to validate the RNA-seq data. As shown in Additional file 3 and Fig. 2, the data for both up- and down-regulated genes from qPCR exhibited excellent agreement with those of RNAseq. In addition, a Spearman bivariate correlation analysis revealed a highly correlated significance $(p<0.01$, correlation coefficient $=0.894$ ) between the data of RNA-seq and qPCR. These data indicate the reliability of RNA-seq data.

\section{Gene ontology (GO) enrichment analysis of genes deregulated during early stages of liver compensatory growth after removing the ventral lobe}

Genes up- and down-regulated at each time point were subjected to GO enrichment analysis in order to identify temporal transcriptional events occurred during the process of liver compensatory growth after hepatectomy of the ventral lobe. The results of GO analysis were displayed in Additional file 4 and representative biological 


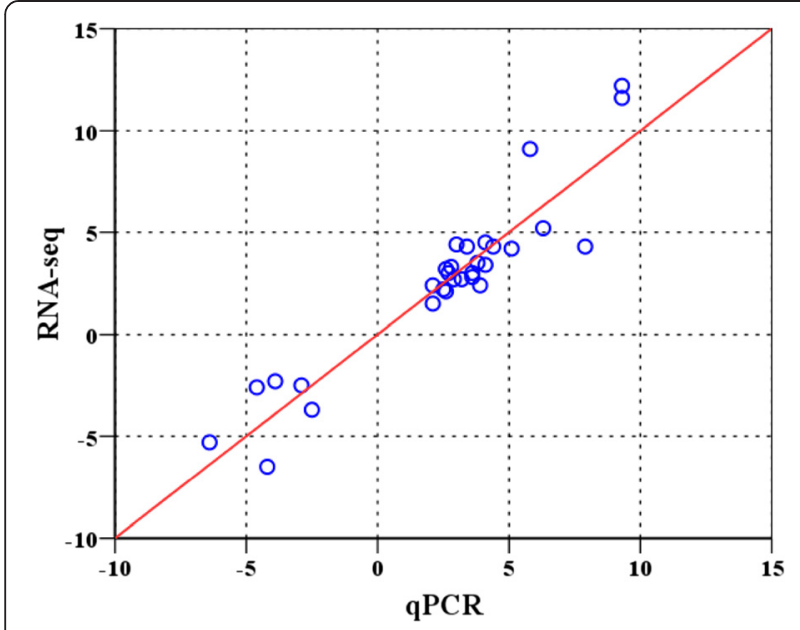

Fig. 2 Validation of RNA-seq data with qPCR. Expression data of genes detected by RNA-seq was plotted against those by qPCR. The reference line indicates the linear correlation between the RNA-seq and qPCR

process terms were displayed in Fig. 3. Overrepresented biological processes of genes particularly up-regulated at 6-h time point after PH include intracellular protein transport, small GTPase mediated signal transduction, RNA processing, gene expression, membrane fusion, regulation of translation, organelle fusion as well as tRNA processing (Fig. 3a). Processes enriched in up-regulated genes at 24-h time point after $\mathrm{PH}$ include tRNA aminoacylation for protein translation, signal peptide processing, regulation of actin cytoskeleton organization, proteolysis, protein polymerization, membrane docking, amino acid activation and fatty acid metabolic process (Fig. 3a). Additionally, protein transport, cellular process, lipid biosynthetic process, response to stress and ATP hydrolysis coupled proton transport, were enriched among up-regulated genes at both 6-h and 24-h time points after removal of the ventral lobe (Additional file 4).

GO analysis of the earliest genes specifically downregulated at 6-h time point after $\mathrm{PH}$ indicated that biological processes such as cellular aldehyde metabolic process, cortical cytoskeleton organization, fatty acid metabolic process, endothelial cell proliferation, and negative regulation of TOR signaling cascade were overrepresented (Fig. 3b). At later time point, biological processes overrepresented among down-regulated genes include tRNA modification and methylation, translational initiation, RNA processing, ribosome biogenesis, regulation of cell proliferation and ncRNA processing (Fig. 3b).

\section{KEGG pathway enrichment analysis of genes deregulated during early stages of liver compensatory growth after hepatectomy of the ventral lobe}

The results of KEGG pathway enrichment analysis were displayed in Additional file 5 and representative biological
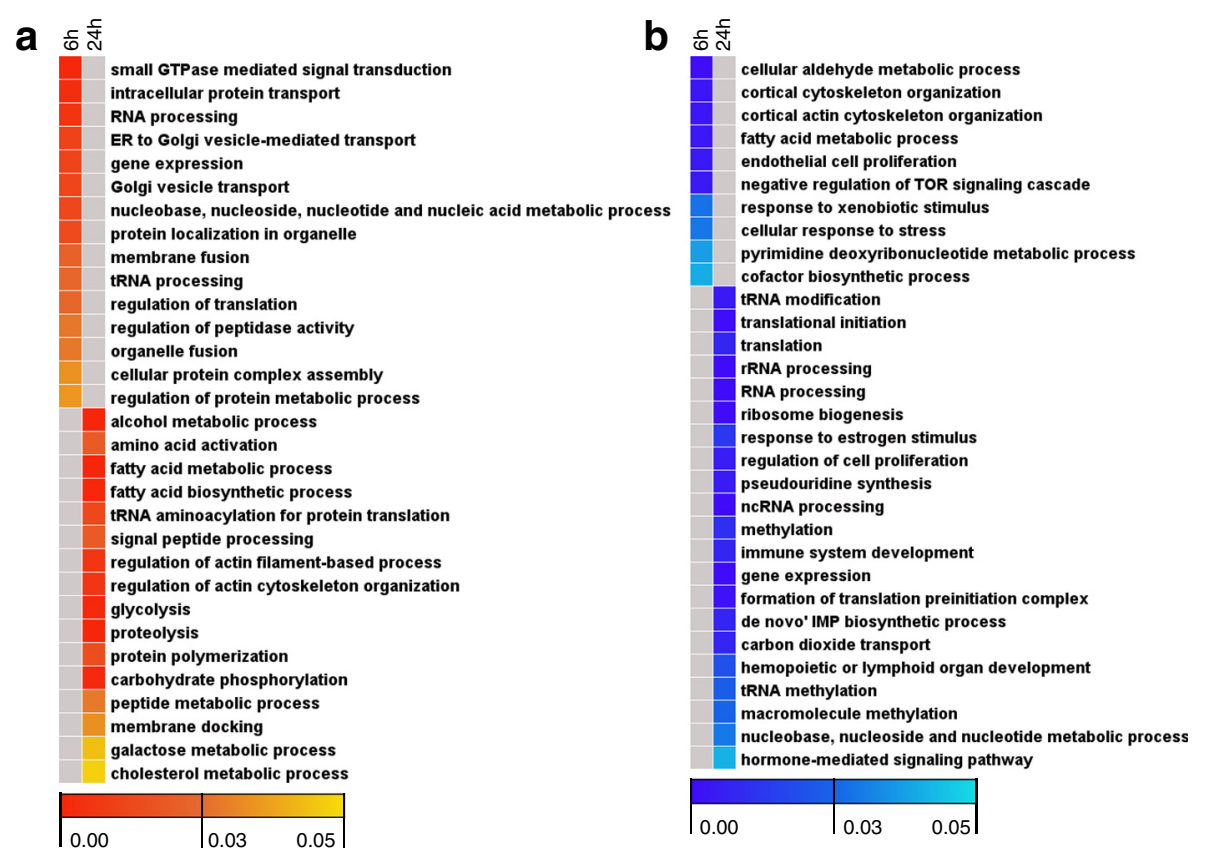

Fig. 3 Heat maps of GO enrichment analysis for liver compensatory growth-associated genes. a Up-regulated genes. b Down-regulated genes. Genes up- or down-regulated at each time point were subjected to GO enrichment analysis for biological processes with BINGO plugin of Cytoscape. GO terms were selected to display in heat maps according to their statistical significance and locations in the GO tree. Columns and rows in the heat maps indicate times after surgery and enriched biological process GO terms, respectively. Sample names were displayed above the heat maps. Color scales indicate $p$-values of enrichment tests and gray cells represent an empty value or a value $>0.05$ 
process terms were displayed in Fig. 4. Pathways including amino sugar and nucleotide sugar metabolism, protein processing in endoplasmic reticulum, proteasome, phagosome, fructose and mannose metabolism, butirosin and neomycin biosynthesis were enriched among up-regulated genes at both 6-h and 24-h time points after PH (Fig. 4a). The earliest pathways overrepresented in genes specifically up-regulated at 6-h time point after surgery include snare interactions in vesicular transport and basal transcription factors. At later time point, pathways overrepresented among up-regulated genes include glycosylphosphatidylinositol (GPI)-anchor biosynthesis, pentose phosphate pathway, glycolysis/gluconeogenesis, starch and sucrose metabolism, galactose metabolism, N-glycan biosynthesis and steroid biosynthesis (Fig. 4a).

In addition, pathways such as retinol metabolism, pyrimidine metabolism, phenylalanine metabolism and propanoate metabolism were overrepresented among down-regulated genes at both 6-h and 24-h time points after surgery (Additional file 5). The earliest pathways overrepresented in genes specifically down-regulated at 6-h time point after $\mathrm{PH}$ include synthesis and degradation of ketone bodies, fatty acid degradation, glutathione metabolism, peroxisome, $\mathrm{ABC}$ transporters, primary bile acid biosynthesis, pantothenate and CoA biosynthesis, glycosaminoglycan degradation and purine metabolism (Fig. 4b). At later time point, pathways such as RNA degradation, cysteine and methionine metabolism, glycerolipid metabolism, tyrosine metabolism and nitrogen metabolism were enriched (Fig. 4b).

SNARE system is central to the sorting, export and recycling of numerous soluble and membrane-associated lysosomal and secretory pathway proteins [34, 35]. We found that SNARE interaction in vesicular transport was the most significantly enriched pathway at 6-h time point but not found at 24-h time point after the ventral lobe removed, in which $25.58 \%$ (11) of associated genes were up-regulated (Additional file 5 and Fig. 5). Moreover, a majority of factors associated with the trans-Golgi network (TGN)-endosomal system, such as Stx1-4 (syntaxin1-4), Stx6 (syntaxin-6), Stx11 (syntaxin-11), Vamp4 (vesicle-associated membrane protein 4) and Snap29 (synaptosomalassociated protein 29), were up-regulated at the 6-h time point during the process of liver compensator growth (Fig. 5).

Furthermore, proteasome was the most highly represented pathway at 24-h time point after hepatectomy of the ventral lobe, in which $64.28 \%$ (36) of associated genes were up-regulated (Additional file 5 and Fig. 6). All the genes encoding proteins in the $20 \mathrm{~S}$ core particle were up-regulated at 24-h time point during the process of liver compensatory growth. Meanwhile, an alternative $\beta$ form denoted $\beta 1 \mathrm{i}$ was also up-regulated at 24 -h time point after PH. Furthermore, a majority of genes associated with the two $19 \mathrm{~S}$ regulatory particles were upregulated at 24-h time point after surgery (Fig. 6).

Thus, these findings suggest that transportation, recycling and degradation of soluble and membrane-associated proteins are actively involved in the process of liver compensator growth.

Comparison of genes differentially expressed during early stages of liver compensatory growth between mice and female zebrafish

Gene expression profiles during early stages of liver compensatory growth in mice and female zebrafish were

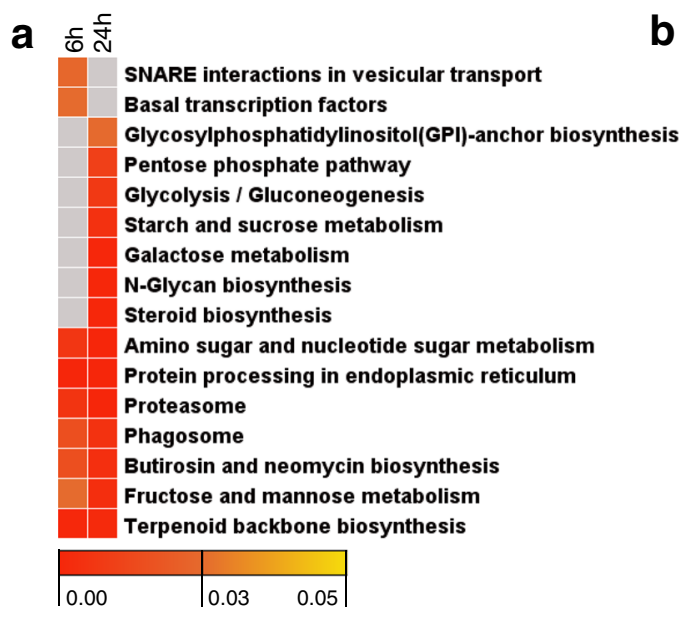

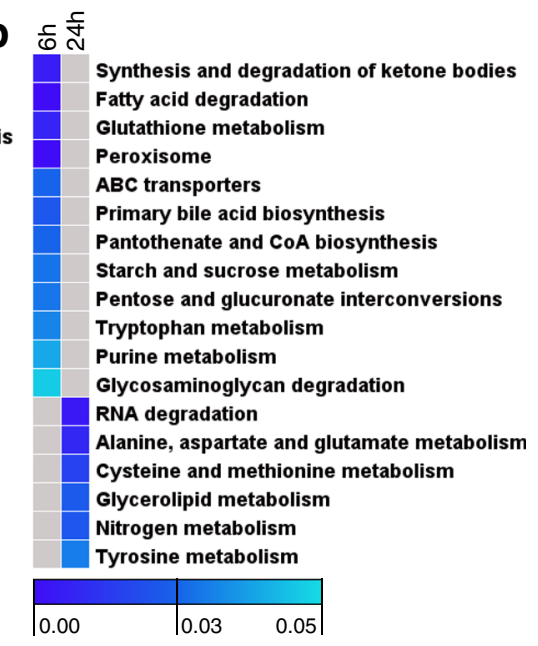

Fig. 4 Heat maps of pathway enrichment analysis for liver compensatory growth -associated genes. a Up-regulated genes. b Down-regulated genes. Genes up- or down-regulated at each time point were subjected to pathway enrichment analysis with KEGG by ClueGO plugin of Cytoscape. Columns and rows in heat maps indicate times after surgery and enriched pathway terms, respectively. Sample names were displayed above the heat maps. Color scales represent $p$-values of enrichment tests and gray cells indicate an empty value or a value $>0.05$ 


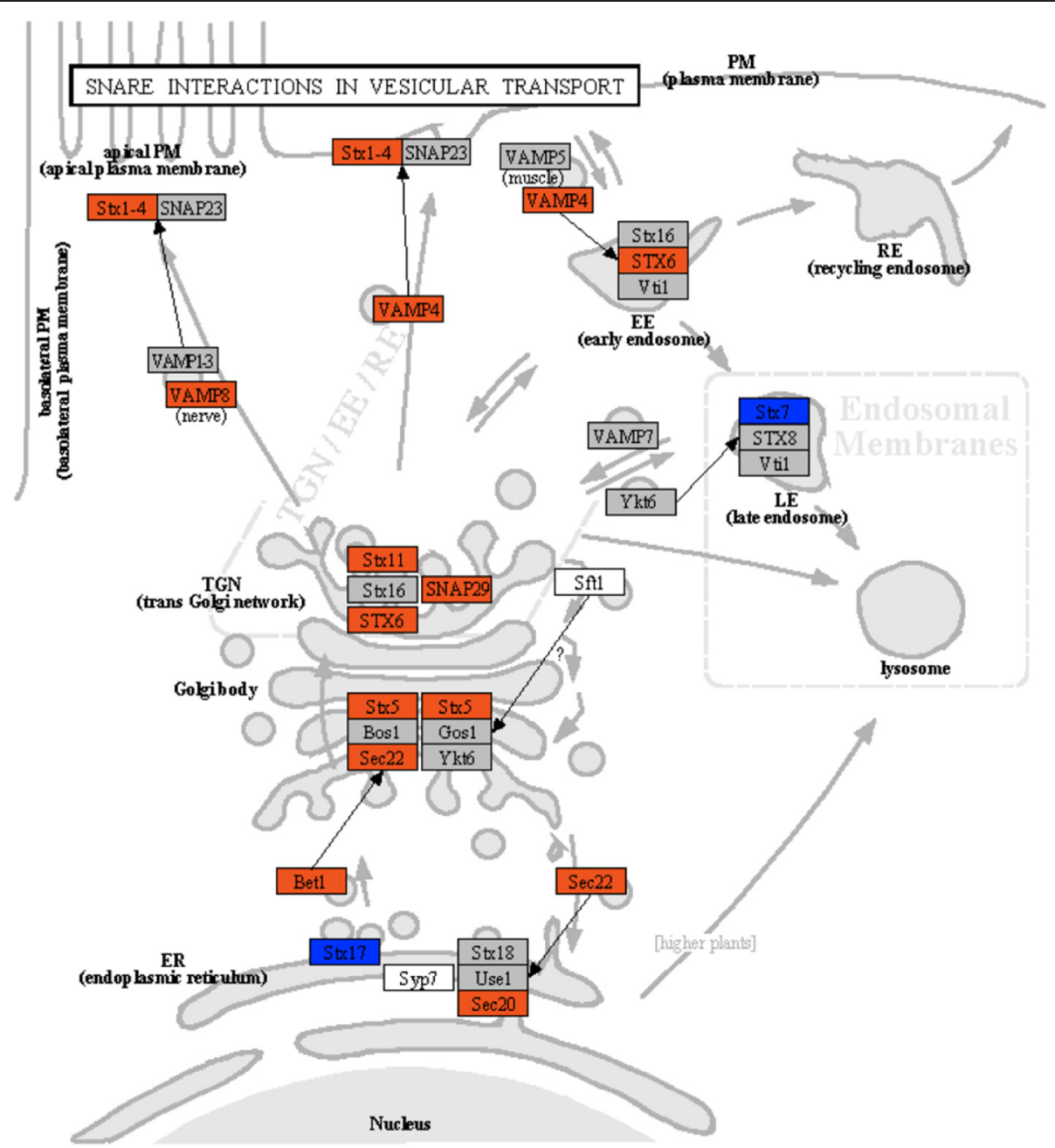

Fig. 5 Some of up-regulated genes at 6-h time point after PH were associated with the SNARE interactions in vesicular transport pathway. Gene expression value was mapped to the reference pathway with the KegArray. Up- and down-regulated genes are shown in orange and blue, respectively

compared to identify factors that are conservatively required for liver compensatory growth in vertebrate organisms. Of 5652 transcripts that are differentially expressed during early stages of liver compensatory growth in female zebrafish, 477 genes were differentially expressed during early stages of this process in mice (Additional file 6). For instance, Angptl4 (angiopoietin-like 4) is up-regulated in both mice and female zebrafish, indicating its important role in liver compensatory growth of both organisms. GO enrichment analysis indicated that most enriched biological processes of these 477 differently expressed genes both in mice and female zebrafish include the response to stimulus, DNA replication, multicellular organismal homeostasis, metabolic processes of fatty acid, lipid and steroid and extracellular matrix constituent secretion (Additional file 7 and Additional file 8), suggesting these biological processes play conservative roles in liver compensatory growth of mice and female zebrafish.

\section{Discussion}

The compensatory growth of liver after hepatectomy of the ventral lobe occurs in zebrafish and this process, similar to those in rodents and humans, is closely associated with the activation and proliferation of hepatocytes [6], but origins of the initial signals for the activation of hepatocytic cells in the remaining liver lobes are largely unknown. In this study, transcriptional expression of genes involved in early stages of liver compensatory growth in female zebrafish was systematically examined by using RNA-seq. Transcriptional profiling of liver dorsal lobes has revealed genes that are differentially expressed at 6 and $24 \mathrm{~h}$ after $\mathrm{PH}$ and some of these genes encode proteins that serve as key components of intracellular signaling pathways in eukaryotes. However, the identification of master genes and key biological processes involved in the initiation of liver compensatory growth after removing the ventral lobe remains a challenging task. 


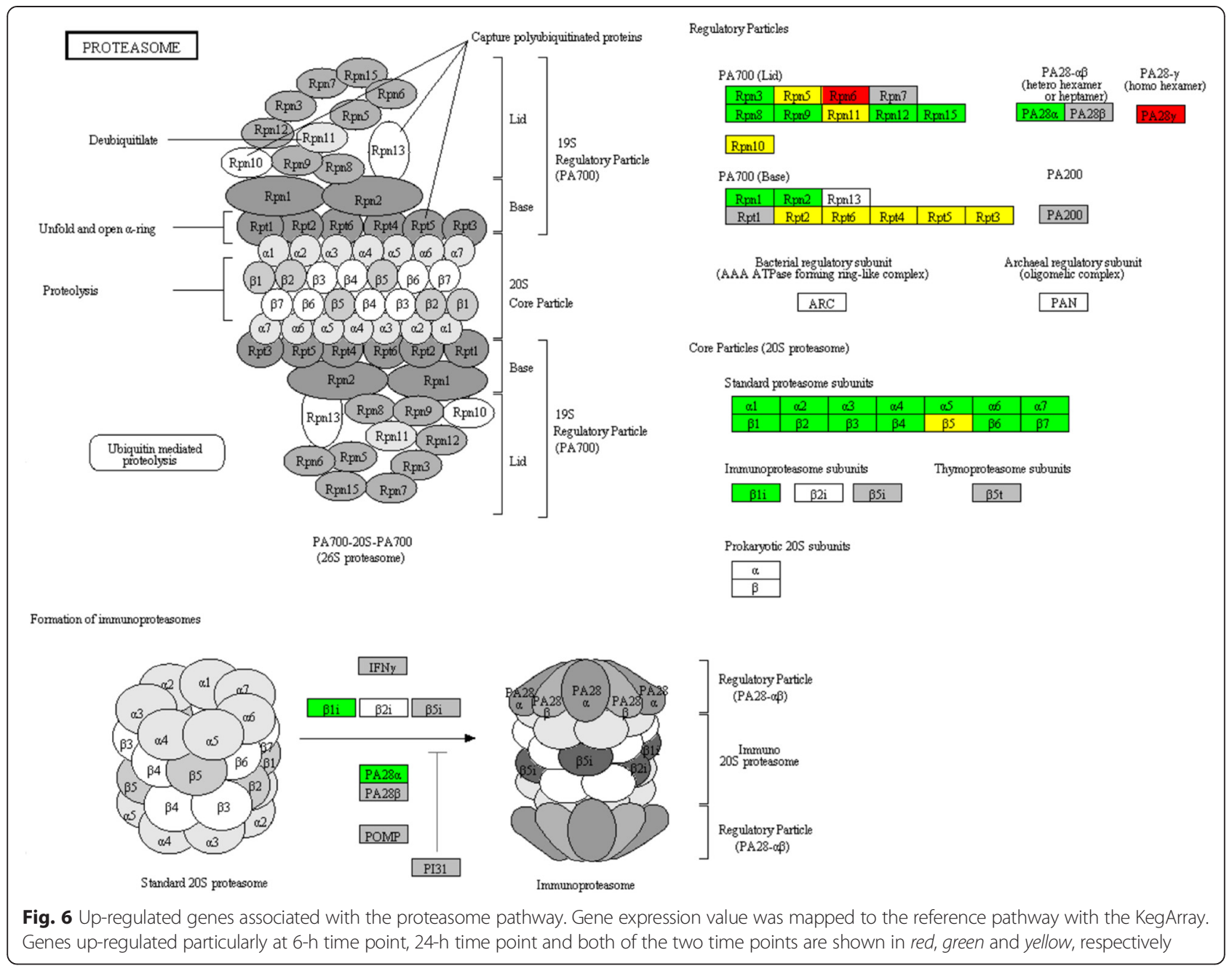

Previous studies have placed the expression patterns of many genes that are either newly expressed or increased after $\mathrm{PH}$ into different stages, including immediate-early genes, delayed genes and cell cycle genes, to facilitate the understanding of the molecular events during the liver compensatory growth [36]. The immediate early stage occurs very rapidly and lasts for approximately $4 \mathrm{~h}$ [36] and the DNA replication and proliferation of hepatocytes after PH start at approximately $24 \mathrm{~h}$ in zebrafish [9]. Thus, we have selected the 6-h and 24-h time points after removal of the whole ventral lobe to uncover molecular events that are essential for the initiation of liver compensatory growth in female zebrafish.

The proliferation of hepatocytes is one of hallmarks in the remaining liver lobes after massive tissue loss [1]. Secreted molecules originated from the circulating blood or adjacent cells in the wound are likely serving as the signals to trigger the activation of cells in the remaining liver, since several genes including egfr, tgfbla, apoeb, $i l 7 r$, il1b, igfbp1, igfbp3, ctgf, angptl2b, angptl4, tnfrsfla and $t$ nfrsf $9 a$, were identified by RNA-seq to encode these kinds of factors or their receptors in this study. EGFR is a critical regulator of hepatocyte proliferation in the initial phases of liver compensatory growth in mice [33]. Mice deficient in IGFBP1 displayed a phenotype of liver necrosis and a reduced and delayed DNA synthesis in hepatocytes after PH [37]. CTGF, also known as CCN2, plays important roles in many of biological processes including cell proliferation, angiogenesis and tissue wound repair $[38,39]$ and its induction is important for robust oval cell response during liver compensatory growth after 2$\mathrm{AAF} / \mathrm{PHx}$ treatment in rats [40]. ANGPTL4 (angiopoietinlike 4) functions as a matricellular protein [41] to stimulate STAT3-mediated iNOS expression, enhance the angiogenesis and accelerate the wound healing in diabetic mice [42]. ANGPTL4-deficient mice exhibit delayed wound reepithelialization with impaired keratinocyte migration, angiogenesis and altered inflammatory response [43, 44].

Several angiogenesis-associated genes including vegfc, vegfaa, cxcr 7, mmp9, mmp13a, mmp17b and itgav were markedly up-regulated in the remaining dorsal lobes. Vegfc is required for vasculogenesis and angiogenesis in 
the zebrafish embryo [45]. Vegf is suggested to play an important role in liver compensatory growth due to its effects on neovascularization in the rat model [46]. CXCR7 can deploy pro-regenerative angiocrine factors and trigger liver compensatory growth through inducing the transcription factor Id1 after acute injury in mice [47]. MMPs can facilitate the growth of new capillaries and hepatic compensatory growth due to their direct effect on extracellular matrix remodeling in rats [48-50]. MMP9-deficient mice show a delayed regenerative response after $70 \%$ hepatectomy [51] and suppression of MMP9 activity attenuates microcirculatory obstruction in monocrotaline-induced acute liver injury [52, 53]. ITGAV (integrin alpha V) can combine with ITGB3 (integrin beta 3 ) and play an important role in vascular repair processes [54]. Therefore, angiogenesis appears to be activated in the initiation of liver compensatory growth in female zebfafish.

Moreover, a few of apoptotic factors encoded by casp3, casp8, casp9, casp8l2, dffa, tp53, baxa, endog, cycsb and traf $2 b$, were identified during the compensatory growth of liver dorsal lobes. It is suggested that apoptosis can be induced indirectly by cells that undergo necrosis in response to overwhelming physical injury and may be a driving force for cell proliferation during tissue regeneration in many different organisms [55]. Casp3 and DFFA are found to be responsible for chromatin condensation and DNA fragmentation during apoptosis [56, 57]. Mice lacking casp3 have impaired wound healing and defects in liver compensatory growth after PH [58]. Casp8 has been implicated in signaling for apoptotic cell death and certain non-apoptotic functions during liver compensatory growth [59]. Thus, cellular apoptosis appears to function in the remodeling of remaining liver structure and in providing signals for the liver compensatory growth after removal of the ventral lobe in female zebrafish.

GO and KEGG pathway enrichment analysis of genes differentially expressed at 6-h and 24-h time points in dorsal lobes has identified a number of biological processes such as SNARE (soluble $\mathrm{N}$-ethylmaleimide-sensitive factor attachment protein receptors) interaction in vesicular transport, proteasome, RNA processing, small GTPase-mediated signal transduction, and signaling pathways including basal transcription factors that are possibly activated by initial signals and factors for the compensatory growth. SNARE proteins belong to a large protein superfamily in yeast and mammalian cells and complexes of SNAREs on opposing membranes were differentially required for membrane fusion within the secretory pathway $[60,61]$. The proteasomes is a multisubunit enzyme complex that contains one $20 \mathrm{~S}$ protein particle and two 195 regulatory particles and plays a central role in the regulation of protein activities in cellcycle progression and apoptosis [62]. The $\beta 1, \beta 2$ and $\beta 5$ subunits were catalytic for proteolysis with three distinct substrate specificities of chymotrypsin-like, trypsin-like and post-glutamyl peptide hydrolase-like [63]. Meanwhile, an alternative $\beta$ form denoted $\beta 1 \mathrm{i}$ was found to express in hematopoietic cells in response to pro-inflammatory signals such as cytokines and required for immunoproteasome assembly [64]. The pre-mRNA molecule undergoes three main modifications included 5' capping, 3' polyadenylation and RNA splicing in the cell nucleus before the RNA is translated, and this process is designed RNA processing [65]. The post-transcriptional modification process plays important roles during liver compensatory growth due to its effects on transcripts stability, alternative splicing and stabilization of heterogeneous nuclear RNA [66]. Thus, multiple biological processes and signaling pathways are involved in early stages of liver compensatory growth in female zebrafish and exhibit a stage-specific and sequential alteration in gene expression during this process. However, further investigations are needed to identify master factors that control the activation of these biological processes and signaling pathways during the liver compensatory growth in zebrafish.

A comparison of gene expression profiles identified 477 genes differently expressed during liver compensatory growth both in mice and female zebrafish. These genes encode proteins that play important roles in some of crucial processes including DNA replication, multicellular organismal homeostasis, metabolic processes of fatty acid, lipid and steroid and extracellular matrix constituent secretion, indicating these biological processes have conservative functions in liver compensatory growth of vertebrates. However, a majority of transcripts are found to be differentially expressed in female zebrafish but not in mice probably due to their differences in living environments and physiological conditions.

\section{Conclusions}

In this study, we have identified some of key genes encoding angiogenesis-related growth factors/ligands (vegfaa, vegfc, apoeb, cxcr7b, itgav, mmps, ctgfa and angptl4) and apoptosis-associated cytokines (casp $3 a$, casp 8 , casp $9, d f f a$, endog and baxa). These factors are potentially involved in the initiation of liver compensatory growth in female zebrafish. Moreover, some of crucial biological processes and intracellular signaling pathways, such as small GTPase-mediated signal transduction, RNA processing, snare interactions in vesicular transport, proteasome and basal transcription factors, are likely to function in liver compensatory growth of female zebrafish. Obviously, these findings have provided novel clues for further investigation of molecular mechanisms underlying the initiation of liver compensatory growth in zebrafish. 


\section{Methods}

\section{Ethics statement}

The animal protocol for this study was approved by the Animal Care and Use Committee of Hubei Province in China and by the Institutional Animal Care and Use Committee of Institute of Hydrobiology (Approval ID: Y21103-1-501).

\section{Zebrafish maintenance, partial hepatectomy (PH) and extraction of RNA}

Healthy $\mathrm{AB}$ line zebrafish weighing $1.8 \pm 0.3 \mathrm{~g}$ and about 8 months of age were obtained from the fish facilities at the Institute of Hydrobiology, Chinese Academy of Sciences and handled according to the standard protocols [67].

The $\mathrm{PH}$ procedure was strictly performed as described previously [6]. The ventral liver lobe was carefully resected at the very base of the lobe, leading to a $30 \%$ PH. Sham-treated animals were subjected to the same procedure without liver resection. Previous studies have shown that not only the expression levels of some housekeeping genes in liver, such as tubal (tubulin alpha 1) and gapdh (glyceraldehyde-3-phosphate dehydrogenase), were significantly higher in females than those in males [68], but also the expression of a large number of liver-produced secretory proteins were enriched in the female fish [69]. Thus, RNA-seq transcriptomic data of regrowing and/or regenerating livers appears to display sexual dimorphism in zebrafish. To eliminate the influences by gender, regenerating liver samples of two dorsal lobes were pooled at 6 and $24 \mathrm{~h}$ after $\mathrm{PH}$ from ten to twelve of regenerating female livers. Similarly, sham-treated liver samples of two dorsal lobes were pooled from twelve female livers at $24 \mathrm{~h}$ after sham surgery.

Total RNA was extracted using TRIZOL reagents from Invitrogen according to the manufacturer's recommendations. The yield and purity of each RNA sample was determined using the NanoDrop 8000 from Thermo Scientific and further assessed with agarose gel electrophoresis.

\section{Library construction and high-throughput sequencing}

RNA library construction and high-throughput sequencing were performed by experts in the Analytical \& Testing Center at Institute of Hydrobiology, Chinese Academy of Sciences (http://www.ihb.ac.cn/fxcszx/) as described previously [30]. Multiplexed libraries were sequenced for $72 \mathrm{bp}$ at both ends using an Illumina Genome Analyzer IIx platform according to the standard Illumina protocols [29]. The sequencing data have been deposited in NCBI Sequence Read Archive (SRA, http://www.ncbi.nlm.nih.gov/Traces/sra) and the accession number is SRP053395.

\section{Data analysis}

Raw reads were first processed with the FASTX-Toolkit to remove the reads of low quality (phred quality $<5$ ). Read mapping, transcript assembly and differential expression analysis were performed as described previously [70, 71]. Briefly, the preprocessed reads were mapped to the genome sequence of zebrafish (Zv9.72) using TopHat (version 2.0.9) [72] with default parameters except "-segment-mismatches 2 " and "-segment-length 36", then the assembled transcripts were merged with the reference annotation (Danio_rerio.Zv9.72.gtf, downloaded from Ensembl) using cuffmerge and differential expression analysis was performed using cuffdiff [73]. Calculation of mapping statistics, sorting and indexing of the read alignment files were performed using SAMtools (version 0.2.0) [74]. The mapping and assembling results were viewed via the IGVtools (version 2.3.31) [75]. Genes with a fold change $\geq 2$ were considered to be differentially expressed. The differentially expressed genes were clustered using cluster 3.0. Clustering results were visualized using JavaTreeview software [76]. Cytoscape (v.3.0.2) plugins [77], BiNGO (v.3.0.2) [78] and ClueGO (v.1.8) [79] were used for GO and KEGG pathway enrichment analysis, respectively. The ontology and annotation files for GO enrichment analysis were downloaded from the gene ontology website (http://www.geneontology.org/) and the database used for KEGG pathway enrichment analysis released on May 27, 2014. Gitools (v.2.1.1) [80] were used for analysis and visualization of genomic data. Gene identifier conversion was performed by g:Profiler web software (http://biit.cs.ut.ee/gprofiler/gconvert.cgi) [81]. RNA-seq data of female zebrafish were compared with those of mice as described previously [82].

\section{Quantitative real time PCR (qPCR)}

The qPCR analysis was performed as described previously [30] to validate the results of RNA-seq. Eef1a1l1 (eukaryotic translation elongation factor 1 alpha 1, like 1) was used as an internal reference for the normalization of gene expression as described previously [68]. The PCR primers were designed using Primer Premier 6.0 software. Primers used for qPCR were list in Additional file 9.

\section{Statistical analysis}

SPSS 15.0 software for windows was used for statistical analysis. The data of gene expression was analyzed by the independent-samples $t$-test. The correlation between the data of RNA-seq and qPCR was analyzed by the Spearman's rho test.

\section{Additional files}

Additional file 1: Genes differentially expressed during the liver compensatory growth and expression profile clustering of these genes. (XLSX $311 \mathrm{~kb})$ 
Additional file 2: List of differentially expressed genes encoding growth factors/ligands and factors associated with angiogenesis and apoptosis. (XLSX $10 \mathrm{~kb}$ )

Additional file 3: Validation of RNA-seq data with qPCR. (XLSX $9 \mathrm{~kb}$ ) Additional file 4: Enriched GO terms for genes differentially expressed during the liver compensatory growth. (XLSX $72 \mathrm{~kb}$ ) Additional file 5: Enriched pathways for genes differentially expressed during the liver compensatory growth. (XLSX $76 \mathrm{~kb}$ ) Additional file 6: Genes differentially expressed during early stages of liver compensatory growth in both mice and female zebrafish. Livers of hepatectomized old or young fish vs. age-matched controls were analyzed at indicated time points. Data are expressed as Fold Difference (FD) vs. untreated young or adult (control) livers, respectively. Differentially expressed genes were selected with DiffScore cutoff $\geq 20$ or $\leq-20$, a q-value of 0.01 and fold-differences cutoff \pm 2 . NS: gene expression with DiffScore cutoff $\geq-20$ or $\leq 20$. (XLSX $94 \mathrm{~kb}$ )

Additional file 7: Enriched GO terms for genes differentially expressed during early stages of liver compensatory growth both in mice and female zebrafish. (XLSX $15 \mathrm{~kb}$ )

Additional file 8: GO enrichment analysis for genes differentially expressed during early stages of liver compensatory growth both in mice and female zebrafish. The size of circles is proportional to the number of genes associated with the GO term. The arrows represent the relationship between parent-child terms. The color scale indicates corrected p-value of enrichment analysis. (PDF $1822 \mathrm{~kb}$ )

Additional file 9: Primers used for qPCR. (XLSX $11 \mathrm{~kb}$ )

\section{Competing interests}

The authors declare that they have no competing interests.

\section{Authors' contributions}

ZC, JP and GF designed the study and prepared the manuscript. GF and YL performed the experiments and data analysis. QL provided experimental materials. All authors read and approved the final manuscript.

\section{Acknowledgements}

This research was supported by grants from the National Basic Research Program of China (\#2012CB944500) and the National Natural Science Foundation of China (\#31171390 to Z. Cui).

\section{Author details}

'The Key Laboratory of Aquatic Biodiversity and Conservation of Chinese Academy of Sciences, Institute of Hydrobiology, Chinese Academy of Sciences, Wuhan 430072 Hubei, China. ${ }^{2}$ University of Chinese Academy of Sciences, Beijing 100049, China. ${ }^{3}$ Zhejiang University, Hangzhou 310058 Zhejiang, China.

Received: 19 August 2015 Accepted: 26 October 2015

Published online: 19 November 2015

\section{References}

1. Fausto N, Campbell JS, Riehle KJ. Liver regeneration. Hepatology. 2006:43(2 Suppl 1):S45-53.

2. Michalopoulos GK, DeFrances MC. Liver regeneration. Science. 1997;276(5309):60-6.

3. Michalopoulos GK, Khan Z. Liver regeneration, growth factors, and amphiregulin. Gastroenterology. 2005;128(2):503-6.

4. Grisham JW. A morphologic study of deoxyribonucleic acid synthesis and cell proliferation in regenerating rat liver - autoradiography with ThymidineH3. Cancer Res. 1962;22(7):842-9.

5. Taub R. Liver regeneration: from myth to mechanism. Nat Rev Mol Cell Biol. 2004;5(10):836-47.

6. Kan NG, Junghans D, Izpisua Belmonte JC. Compensatory growth mechanisms regulated by BMP and FGF signaling mediate liver regeneration in zebrafish after partial hepatectomy. FASEB J. 2009;23(10):3516-25.
7. Higgins GM, Anderson RM. Experimental pathology of the liver I Restoration of the liver of the white rat following partial surgical removal. Arch Pathol. 1931;12(2):186-202.

8. Korzh S, Pan X, Garcia-Lecea M, Winata CL, Pan X, Wohland T, et al. Requirement of vasculogenesis and blood circulation in late stages of liver growth in zebrafish. BMC Dev Biol. 2008;8:84.

9. Zhu Z, Chen J, Xiong JW, Peng J. Haploinsufficiency of Def activates p53-dependent TGFbeta signalling and causes scar formation after partial hepatectomy. PLoS One. 2014;9(5):e96576.

10. Michalopoulos GK. Liver regeneration. J Cell Physiol. 2007;213(2):286-300.

11. Sakamoto T, Liu ZJ, Murase N, Ezure T, Yokomuro S, Poli V, et al. Mitosis and apoptosis in the liver of interleukin-6-deficient mice after partial hepatectomy. Hepatology. 1999;29(2):403-11.

12. Weglarz TC, Sandgren EP. Timing of hepatocyte entry into DNA synthesis after partial hepatectomy is cell autonomous. Proc Natl Acad Sci U S A. 2000;97(23):12595-600.

13. Ding BS, Nolan DJ, Butler JM, James D, Babazadeh AO, Rosenwaks Z, et al. Inductive angiocrine signals from sinusoidal endothelium are required for liver regeneration. Nature. 2010;468(7321):310-5.

14. Hu J, Srivastava K, Wieland M, Runge A, Mogler C, Besemfelder E, et al. Endothelial cell-derived angiopoietin-2 controls liver regeneration as a spatiotemporal rheostat. Science. 2014;343(6169):416-9.

15. LeCouter J, Moritz DR, Li B, Phillips GL, Liang XH, Gerber HP, et al. Angiogenesis-independent endothelial protection of liver: role of VEGFR-1. Science. 2003;299(5608):890-3.

16. Webber EM, Bruix J, Pierce RH, Fausto N. Tumor necrosis factor primes hepatocytes for DNA replication in the rat. Hepatology. 1998;28(5):1226-34.

17. Date M, Matsuzaki K, Matsushita M, Tahashi Y, Furukawa F, Inoue K. Modulation of transforming growth factor beta function in hepatocytes and hepatic stellate cells in rat liver injury. Gut. 2000;46(5):719-24.

18. Fausto N, Riehle KJ. Mechanisms of liver regeneration and their clinical implications. J Hepato-Biliary-Pancreat Surg. 2005;12(3):181-9.

19. Song G, Sharma AD, Roll GR, Ng R, Lee AY, Blelloch RH, et al. MicroRNAs control hepatocyte proliferation during liver regeneration. Hepatology. 2010;51(5):1735-43.

20. Wuestefeld T, Pesic M, Rudalska R, Dauch D, Longerich T, Kang TW, et al. A Direct in vivo RNAi screen identifies MKK4 as a key regulator of liver regeneration. Cell. 2013;153(2):389-401.

21. Fimbel SM, Montgomery JE, Burket CT, Hyde DR. Regeneration of inner retinal neurons after intravitreal injection of ouabain in zebrafish. J Neurosci. 2007;27(7):1712-24

22. Poss KD, Wilson LG, Keating MT. Heart regeneration in zebrafish. Science. 2002;298(5601):2188-90.

23. Lieschke GJ, Currie PD. Animal models of human disease: zebrafish swim into view. Nat Rev Genet. 2007:8(5):353-67.

24. Chang C, Xu C. Transcriptome atlas of aromatic amino acid family metabolism-related genes in eight liver cell types uncovers the corresponding metabolic pathways in rat liver regeneration. Int J Biochem Cell Biol. 2010;42(10):1708-16.

25. White P, Brestelli JE, Kaestner KH, Greenbaum LE. Identification of transcriptional networks during liver regeneration. J Biol Chem. 2005;280(5):3715-22.

26. Nygard IE, Mortensen KE, Hedegaard J, Conley LN, Kalstad T, Bendixen C, et al. The genetic regulation of the terminating phase of liver regeneration. Comp Hepatol. 2012;11(1):3.

27. Cimica $V$, Batusic $D$, Chen $Y$, Hollemann $T$, Pieler T, Ramadori $G$. Transcriptome analysis of rat liver regeneration in a model of oval hepatic stem cells. Genomics. 2005:86(3):352-64.

28. Wang Z, Gerstein M, Snyder M. RNA-Seq: a revolutionary tool for transcriptomics. Nat Rev Genet. 2009;10(1):57-63.

29. Wang Z, Fang B, Chen J, Zhang X, Luo Z, Huang L, et al. De novo assembly and characterization of root transcriptome using Illumina paired-end sequencing and development of cSSR markers in sweet potato (Ipomoea batatas). BMC Genomics. 2010;11:726.

30. Long Y, Song G, Yan J, He X, Li Q, Cui Z. Transcriptomic characterization of cold acclimation in larval zebrafish. BMC Genomics. 2013;14:612.

31. Mastrodonato M, Portincasa P, Mentino D, Rossi R, Resta L, Ferri D, et al. Caveolin-1 involved in mitochondrial homeostasis and lipidogenesis during rat liver regeneration following partial hepatectomy. Eur J Clin Invest. 2012:42:87-8

32. Fernandez MA, Albor C, Ingelmo-Torres M, Nixon SJ, Ferguson C, Kurzchalia $\mathrm{T}$, et al. Caveolin-1 is essential for liver regeneration. Science. 2006;313(5793):1628-32. 
33. Natarajan A, Wagner B, Sibilia M. The EGF receptor is required for efficient liver regeneration. Proc Natl Acad Sci U S A. 2007;104(43):17081-6.

34. Rothman JE, Wieland FT. Protein sorting by transport vesicles. Science. 1996;272(5259):227-34

35. Traub LM, Kornfeld S. The trans-Golgi network: a late secretory sorting station. Curr Opin Cell Biol. 1997;9(4):527-33.

36. Fausto N. Liver regeneration. J Hepatol. 2000;32(1 Suppl):19-31.

37. Leu JI, Crissey MA, Craig LE, Taub R. Impaired hepatocyte DNA synthetic response posthepatectomy in insulin-like growth factor binding protein 1-deficient mice with defects in C/EBP beta and mitogen-activated protein kinase/extracellular signal-regulated kinase regulation. Mol Cell Biol. 2003;23(4):1251-9.

38. Jun Jl, Lau LF. Taking aim at the extracellular matrix: CCN proteins as emerging therapeutic targets. Nat Rev Drug Discov. 2011;10(12):945-63.

39. Hall-Glenn F, Lyons KM. Roles for CCN2 in normal physiological processes. Cell Mol Life Sci. 2011;68(19):3209-17.

40. Pi LY, Oh SH, Shupe T, Petersen BE. Role of connective tissue growth factor in oval cell response during liver regeneration after 2-AAF/PHx in rats. Gastroenterology. 2005;128(7):2077-88.

41. Chong HC, Tan CK, Huang RL, Tan NS. Matricellular proteins: a sticky affair with cancers. J Oncol. 2012;2012:351089.

42. Chong HC, Chan JS, Goh CQ, Gounko NV, Luo B, Wang X, et al. Angiopoietinlike 4 stimulates STAT3-mediated iNOS expression and enhances angiogenesis to accelerate wound healing in diabetic mice. Mol Ther. 2014;22(9):1593-604.

43. Goh YY, Pal M, Chong HC, Zhu P, Tan MJ, Punugu L, et al. Angiopoietin-like 4 interacts with integrins beta1 and beta5 to modulate keratinocyte migration. Am J Pathol. 2010;177(6):2791-803.

44. Goh YY, Pal M, Chong HC, Zhu P, Tan MJ, Punugu L, et al. Angiopoietin-like 4 interacts with matrix proteins to modulate wound healing. J Biol Chem. 2010;285(43):32999-3009.

45. Ober EA, Olofsson B, Makinen T, Jin SW, Shoji W, Koh GY, et al. Vegfc is required for vascular development and endoderm morphogenesis in zebrafish. Embo Rep. 2004;5(1):78-84.

46. Bockhorn M, Goralski M, Prokofiev D, Dammann P, Grunewald P, Trippler M, et al. VEGF is important for early liver regeneration after partial hepatectomy. J Surg Res. 2007;138(2):291-9.

47. Ding BS, Cao ZW, Lis R, Nolan DJ, Guo PP, Simons M, et al. Divergent angiocrine signals from vascular niche balance liver regeneration and fibrosis. Nature. 2014;505(7481):97-102.

48. Moses MA. The regulation of neovascularization of matrix metalloproteinases and their inhibitors. Stem Cells. 1997;15(3):180-9.

49. Haas TL, Milkiewicz M, Davis SJ, Zhou AL, Egginton S, Brown MD, et al. Matrix metalloproteinase activity is required for activity-induced angiogenesis in rat skeletal muscle. Am J Physiol Heart Circ Physiol. 2000;279(4):H1540-7.

50. Kim TH, Mars WM, Stolz DB, Michalopoulos GK. Expression and activation of pro-MMP-2 and pro-MMP-9 during rat liver regeneration. Hepatology. 2000;31(1):75-82.

51. Olle EW, Ren X, McClintock SD, Warner RL, Deogracias MP, Johnson KJ, et al. Matrix metalloproteinase-9 is an important factor in hepatic regeneration after partial hepatectomy in mice. Hepatology. 2006;44(3):540-9.

52. Periasamy S, Hsu DZ, Chen SY, Yang SS, Chandrasekaran VR, Liu MY. Therapeutic sesamol attenuates monocrotaline-induced sinusoidal obstruction syndrome in rats by inhibiting matrix metalloproteinase-9. Cell Biochem Biophys. 2011;61(2):327-36.

53. Nakamura K, Hatano E, Narita M, Miyagawa-Hayashino A, Koyama Y, Nagata $\mathrm{H}$, et al. Sorafenib attenuates monocrotaline-induced sinusoidal obstruction syndrome in rats through suppression of JNK and MMP-9. J Hepatol. 2012;57(5):1037-43.

54. Sajid M, Stouffer GA. The role of alpha(v)beta3 integrins in vascular healing Thromb Haemost. 2002;87(2):187-93.

55. Bergmann A, Steller H. Apoptosis, stem cells, and tissue regeneration. Sc Signal. 2010. doi:10.1126/scisignal.3145.

56. Porter AG, Janicke RU. Emerging roles of caspase-3 in apoptosis. Cell Death Differ. 1999;6(2):99-104

57. Liu X, Zou H, Slaughter C, Wang X. DFF, a heterodimeric protein that functions downstream of caspase-3 to trigger DNA fragmentation during apoptosis. Cell. 1997;89(2):175-84.

58. Li F, Huang Q, Chen J, Peng Y, Roop DR, Bedford JS, et al. Apoptotic cells activate the "phoenix rising" pathway to promote wound healing and tissue regeneration. Sci Signal. 2010. doi:10.1126/scisignal.2000634.
59. Freimuth J, Bangen JM, Lambertz D, Hu W, Nevzorova YA, Sonntag R, et al. Loss of caspase- 8 in hepatocytes accelerates the onset of liver regeneration in mice through premature nuclear factor kappa B activation. Hepatology. 2013:58(5):1779-89.

60. Hay JC, Scheller RH. SNAREs and NSF in targeted membrane fusion. Curr Opin Cell Biol. 1997;9(4):505-12.

61. Rothman JE. Mechanisms of intracellular protein transport. Nature. 1994;372(6501):55-63.

62. Adams J. The proteasome: structure, function, and role in the cell. Cancer Treat Rev. 2003;29 Suppl 1:3-9.

63. Heinemeyer W, Fischer M, Krimmer T, Stachon U, Wolf DH. The active sites of the eukaryotic $20 \mathrm{~S}$ proteasome and their involvement in subunit precursor processing. J Biol Chem. 1997:272(40):25200-9.

64. Nandi D, Tahiliani P, Kumar A, Chandu D. The ubiquitin-proteasome system. J Biosci. 2006;31(1):137-55.

65. Lodish HF BA, Kaiser C, Krieger M, Scott MP, Bretscher A, Ploegh H, Matsudaira PT. Chapter 8: Post-transcriptional Gene Control. In: Freeman W, editor. Molecular Cell Biology. San Francisco; 2007.

66. Kren BT, Steer CJ. Posttranscriptional regulation of gene expression in liver regeneration: role of mRNA stability. FASEB J. 1996;10(5):559-73.

67. Westerfield M. The zebrafish book : a guide for the laboratory use of zebrafish (Brachydanio rerio). Eugene: M. Westerfield; 1993.

68. McCurley AT, Callard GV. Characterization of housekeeping genes in zebrafish: male-female differences and effects of tissue type, developmental stage and chemical treatment. BMC Mol Biol. 2008;9:102.

69. Wen C, Zhang Z, Ma W, Xu M, Wen Z, Peng J. Genome-wide identification of female-enriched genes in zebrafish. Dev Dyn. 2005;232(1):171-9.

70. Trapnell C, Roberts A, Goff L, Pertea G, Kim D, Kelley DR, et al. Differential gene and transcript expression analysis of RNA-seq experiments with TopHat and Cufflinks. Nat Protoc. 2012;7(3):562-78.

71. Long Y, Yan J, Song G, Li X, Li X, Li Q, et al. Transcriptional events co-regulated by hypoxia and cold stresses in Zebrafish larvae. BMC Genomics. 2015;16:385.

72. Trapnell C, Pachter L, Salzberg SL. TopHat: discovering splice junctions with RNA-Seq. Bioinformatics. 2009;25(9):1105-11.

73. Trapnell C, Williams BA, Pertea G, Mortazavi A, Kwan G, van Baren MJ, et al. Transcript assembly and quantification by RNA-Seq reveals unannotated transcripts and isoform switching during cell differentiation. Nat Biotechnol. 2010;28(5):511-5

74. Li H, Handsaker B, Wysoker A, Fennell T, Ruan J, Homer N, et al. The Sequence Alignment/Map format and SAMtools. Bioinformatics. 2009;25(16):2078-9.

75. Thorvaldsdottir H, Robinson JT, Mesirov JP. Integrative Genomics Viewer (IGV): high-performance genomics data visualization and exploration. Brief Bioinform. 2013:14(2):178-92

76. Saldanha AJ. Java Treeview-extensible visualization of microarray data. Bioinformatics. 2004;20(17):3246-8.

77. Shannon P, Markiel A, Ozier O, Baliga NS, Wang JT, Ramage D, et al. Cytoscape: a software environment for integrated models of biomolecular interaction networks. Genome Res. 2003;13(11):2498-504.

78. Maere S, Heymans K, Kuiper M. BiNGO: a Cytoscape plugin to assess overrepresentation of gene ontology categories in biological networks. Bioinformatics. 2005;21(16):3448-9.

79. Bindea G, Mlecnik B, Hackl H, Charoentong P, Tosolini M, Kirilovsky A, et al. ClueGO: a Cytoscape plug-in to decipher functionally grouped gene ontology and pathway annotation networks. Bioinformatics. 2009;25(8):1091-3

80. Perez-Llamas C, Lopez-Bigas N. Gitools: analysis and visualisation of genomic data using interactive heat-maps. PLoS One. 2011;6(5):e19541.

81. Reimand J, Kull M, Peterson H, Hansen J, Vilo J. g:Profiler-a web-based toolset for functional profiling of gene lists from large-scale experiments. Nucleic Acids Res. 2007;35(Web Server issue):W193-200.

82. Pibiri M, Sulas $\mathrm{P}$, Leoni VP, Perra A, Kowalik MA, Cordella A, et al. Global gene expression profile of normal and regenerating liver in young and old mice. Age. 2015;37(3):9796 